\title{
Syntactic Pattern Recognition Using Finite Inductive Strings
}

\author{
Paul Fisher ${ }^{1}$, Howard Fisher ${ }^{2}$, Jinsuk Baek ${ }^{1}$, and Cleopas Angaye ${ }^{3}$ \\ ${ }^{1}$ Department of Computer Science, Winston-Salem State University, \\ Winston-Salem, North Carolina, United States \\ ${ }^{2}$ Fisher Company, Salt Lake City, Utah, United States \\ ${ }^{3}$ National Information Technology Development Agency, Nigeria, Africa
}

\begin{abstract}
A syntactic pattern recognition technique is described based upon a mathematical principle associated with finite sequences of symbols. The technique allows for fast recognition of patterns within strings, including the ability to recognize expected symbols that are close to the desired symbols, and mutations as well as both local and global substring matching. This allowance of deviation permits sequences to be subject to error and still be recognized. Some examples are provided illustrating the technique.
\end{abstract}

Keywords: Pattern recognition, finite inductive sequences, syntactic pattern recognition, genome recognition.

\section{Introduction}

We provide a description including the properties of finite inductive sequences, and two programs which utilize this formulation to process strings of unknown patterns for recognition of subsequences of symbols that occur within other sequences. There have been and continue to be syntax pattern recognition systems for application within the biomedical field, as well as utilization in many other fields.

The basis for this work began many years ago with the consideration of sequences of control structures for autonomous robots. Needing a concise and simple way of representing learned activities, and using that learned experience to drive the next steps, we proposed the concept of Finite Inductive Sequences. When experienced as next activities, the results would provide some measure of new data that would be incorporated into the 'learned' model. So in either stationary models where the rules do not evolve, thus less interesting, or non-stationary models where the rules would be altered to adapt to the changing conditions, provided the impetus to find a solution [3].

The need for more efficient pattern detection algorithms is especially urgent in bioinformatics where genome-wide association studies (GWASs) are becoming increasingly popular in determining the relationship between genetic variations such as single nucleotide polymorphisms (SNPs) and copy-number variations (CVNs) and disease. Current software tools used to analyze these large datasets are computationally intensive. The strength of the finite inductive method is in its ability to determine small variations over a large data set. We believe that this algorithm is ideally suited to improve the speed of GWASs. We note that many tools exist for processing the 
genome sequences, and new applications using graphical display, color, 3D and other extensions have and are being developed [2].

At issue is both speed and accuracy with sufficient flexibility to deal with insertions and deletions in the sequence comparison processes. This has given birth to extensions of the dynamic programming approach first developed for the longest common subsequence (LCS) problem using modifications to the Smith-Waterman algorithm using both hardware and software systems [1], [5], [6], [7], [8], [10]. These systems utilize algorithms ranging over neural nets, Bayesian statistics, tree structures and many other approaches incorporating video algorithms, visualization, data mining, etc., and each has been used in the analysis and recognition process for both protein and amino acid sequences. One only need look at any conference or journal to find articles detailing such research and development activities. All implemented systems must incorporate work from many aspects of science to accomplish the continued analysis of the data which arrives at a rate estimated to be two-million DNA bases each day [8].

We take some space to describe this algorithm which we propose for the purpose of subsequence processing, and indicate some extensions this approach provides. We also provide a measure of the storage and processing complexity of this approach, and lastly, we provide some small examples illustrating the application of FI to the genomic problems.

\section{Finite Inductive Sequences}

In the next sections we describe first a sequence of symbols ('string') which is finitely inductive. Following, that we provide a description of the algorithm for building a set of rules from exemplar sequences together with their associated storage structure called a ruling. Lastly, we show how these rules can be used to recognize patterns in similar types of sequences. Here similar implies that all sequences are formed from identical alphabets.

\subsection{Definition of Finite Inductive Sequences}

In this section, we will describe a particular kind of sequence composed of symbols coming from some alphabet. Consider a sequence of symbols made up from some alphabet such as the representation of the genome with alphabet A, C, G and T. The technique which we shall describe resembles neural net capabilities [9] and emulates Markov models, but with some important differences to both approaches. Consider one or more sequences of symbols, and we can be process them as a single entity or as multiple strings representing data from several sources to be processed in parallel. These sequences we have termed Finitely Inductive Sequences (FI), and such sequences form a countable collection of sequences of finite length. (We will not address infinite sequences, although there is a class of such infinite sequences that are amenable to this approach.) FI has a formal background in mathematics and lies between a mathematical characterization, on the one hand, and a statistical characterization, on the other. The idea is generally to deal with sequences or subsequences observed previously- such as might have been seen previously or learned through exemplar sequences-then such 
sequences can be used to recognize new, similar subsequences from other strings that one desires to process. Unlike neural nets, it is additionally possible to dynamically modify the data structure formed from exemplar sequences so that new experience can be acquired in near real time from new data sequences.

The primary data structure of FI, called a ruling, is a finite state machine that can use a short driving sequence to generate another sequence that may be much longer, or it can be used in reverse to produce a residual short sequence from a presented long sequence. The residual is the 'left over' symbols from the string that are not part of the experience base encapsulated within the ruling. In a sense, the size of the residual is a measure of the common experience between an unknown event sequence and the string(s) placed in the ruling. The ruling embodies patterns of interest, and acts as a filter through which unknown strings can be filtered looking for identical or similar subsequences found within the ruling and the unknown string.

Finite sequences of symbols are said to be finitely inductive (FI) over a finite alphabet if the choice of any symbol at any particular position in a sequence depends immediately upon only the choices of symbols at the previous $n$ points (the similarity to Markov models except this is generally deterministic and not probablistic.) Given an FI sequence (deterministic), an implicant is a pair $(w, p)$ consisting of a word $w$ over the alphabet and a symbol $p$ of the alphabet such that $w$ occurs at least once as a substring of the sequence; and whenever $w$ occurs as a sub-string of the sequence there is a succeeding entry and it is $p$. For non-deterministic implicants, there might be two or more values for $p$ with a probability associated with each such value. The antecedent is $w$ while $p$ is called the consequent. A reduced form implicant has no proper segment of its antecedent in another implicant.

We note the following:

a. Every finite sequence is finitely inductive.

b. For any finitely inductive sequence, the inductive base is the maximal length of the antecedents when considering all of its reduced form implicants.

c. If an FI sequence has inductive base $n$ and an alphabet of $k$ symbols, then $k^{n}$ is an upper bound for the number of its reduced form implicants.

\subsection{Description of the Storage Structure for Finite Inductive Sequences}

Figure 1 is an example of function table (called a Ruling) resulting from an FI sequence(s) meeting the requirement for an optimal storage system for streams of symbols. The system consists of a structure with $k$ levels. The rules are defined from the exemplar patterns according to a push up formulation of the strings. The inductive base in Figure 1 is as defined above and is equivalent to the longest antecedent in reduced form. The rules are formulated much like those characterizing a Markov process. (We point out another difference between Markov Models and FI sequences is that within the theory of FI sequences; the order of the sequence, i.e., its inductive base, can be altered to any a priori value desired, unlike the order of the Markov Model.)

Given some number $n$, the inductive base, and a sequence of symbols, then we can say that the previous $n$ characters uniquely determine the next character in the string. As the inductive base is reduced, the number of levels will increase. The number of rules remains nearly invariant as the number of levels change. The last three elements in Figure 1, the rules, starting sequence, and the shift register will be explained by an example. 


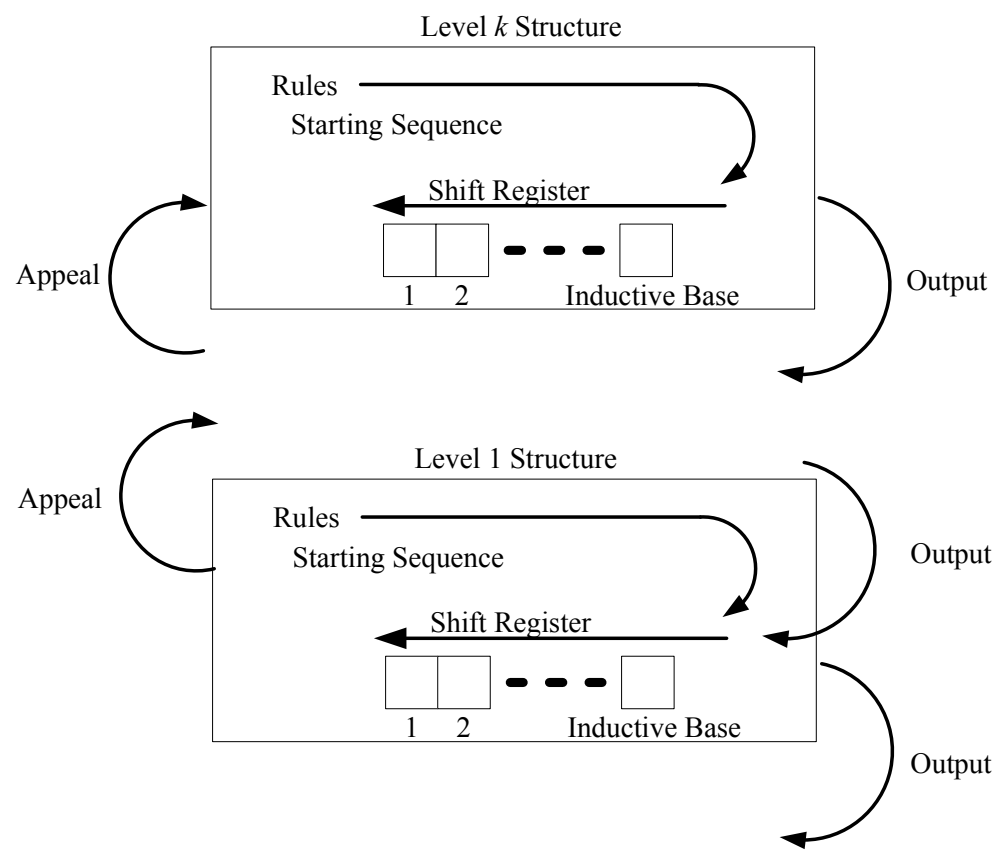

Fig. 1. The FI Ruling Structure for Representing a Sequence(s) in $k$-Levels

Consider the following string to build the structure of Figure 1. We note that the starting symbol ' $\mathrm{S}$ ' is not part of the sequence, but it allows a simpler representation.

\section{S2446664882443}

This sequence comes from an alphabet of eight symbols: $1,2,3,4,5,6,7,8$. It is finite in length, and therefore is finitely inductive. We can form rules for this sequence as a function of the inductive base. However, since the inductive base must be large enough to uniquely guarantee the next symbol, an inductive base of two or less would be unsuitable since the occurrence of ' 66 ' does not uniquely specify the next symbol ('66' precedes both a '6' and an '4'). We can easily extend this representation to the non-deterministic case and to a probabilistic case by specifying that the sequence 66 specifies both a ' 6 ' and a ' 4 ', and if we associate a probability with the two alternatives, then we have the probabilistic representation. We illustrate the table that is generated for the reduced form rules. The first rule in (2) states that each time we see the antecedent ' $S$ ' then a ' 2 ' follows, without ambiguity, as the next symbol in the string.

$$
\begin{aligned}
& \mathrm{S} \rightarrow 2, \quad \mathrm{~S} 2 \rightarrow 4, \quad 24 \rightarrow 4, \quad \mathrm{~S} 244 \rightarrow 6,8244 \rightarrow 3 \quad 46 \rightarrow 6, \\
& 466 \rightarrow 6, \quad 666 \rightarrow 4, \quad 64 \rightarrow 8, \quad 48 \rightarrow 8 \quad 88 \rightarrow 2, \quad 82 \rightarrow 4
\end{aligned}
$$

From (2) the inductive base is 4 (the largest of the antecedent values). Some of the rules are inductive base 1 and 2; when this variability occurs, we can reduce the inductive base (the 3 ) to any value less than the 3 . Suppose we wish to use an inductive 
base of 2, we would get the FI structure of Figure 1, and the process (called Factoring) for deriving this new FI ruling structure is shown in Example 1.

Example 1. Simple String Factoring and Regeneration

For the string: S2446664882443 using the reduced rules (2), define the ruling as follows:

Step 1: Keep all rules shown in (2) with inductive base 2 or less. For everything else push the consequent symbol up out of the string to the next level, which we designate Level 1. This results in the following pattern:

Level $1 \quad \mathrm{~S} \quad 6 \quad 64 \quad 3$

Level $0 \quad$ S2446664882443

The Rules for Level 0 are those representing all symbols not pushed up:

$$
\mathrm{S} \rightarrow 2 \quad \mathrm{~S} 2 \rightarrow 4 \quad 24 \rightarrow 4 \quad 46 \rightarrow 6 \quad 64 \rightarrow 8 \quad 48 \rightarrow 8 \quad 88 \rightarrow 2 \quad 82 \rightarrow 4
$$

Step 2: For the string of Level 1, we find the reduced rules. These are:

$$
\mathrm{S} \rightarrow 6 \quad \mathrm{~S} 6 \rightarrow 6 \quad 66 \rightarrow 4 \quad 4 \rightarrow 3
$$

This process has produced rules of inductive base less than or equal to 2 , in a ruling of two levels, and since there are no symbols that need to be processed further, we stop the process. We also point out the symbols in Level 1 are called the residual from Level 0 . As noted, there is no residual from Level 1. We also point out that every FI string of finite length produces a ruling where the last level has no residual, when that string is processed independently of any other string.

The inductive base for the sequence is 2 . The starting sequences are loaded in the shift registers, and the system is ready to regenerate the original sequence. This is done in the following steps:

Step 1: The $S$ in the shift register of Level 0 is matched against the antecedents of the rules in that level. In this case the antecedent of the string $S \rightarrow 2$ matches the character in the Shift Register.

Step 2: Push the consequent '2' into the right side of the shift register.

Step 3: Since the shift register now contains 'S2', see whether this string or the symbol '2' (top of the shift register) matches any antecedent in this level. There is a match, so the consequent ' 4 ' is placed in the top of the Shift Register, and the ' $\mathrm{S}$ ' is output.

Step 4: Again the Shift Register content of '24' is matched to the antecedents of Level 0 . A match is found, and the consequent ' 4 ' is pushed into the shift register while the ' 2 ' is pushed out to the output.

Step 5: The Level 0 Shift Register now contains '44', and this does not match any of the antecedents in Level 0, so an appeal is made to Level 1. The current status is now:

Level 1: shift register: $\mathrm{S}$

Level 0: shift register: 44 and the output S2 has been generated from level 1. 
Step 6: In Level 1, the contents of the Shift Register are matched, and the consequent ' 6 ' is placed in the shift register, and the ' 6 ' is pushed down to Level 0 and placed in the top of the Shift Register for that level. Now we have the following configuration:

Level 1: Shift Register: '6'

Level 0: Shift Register: '46'

Output: 'S24'

Step 7: Continue with processing in Level 0 until no processing can continue, and then output the Shift Register of Level 0 to complete the original sequence regeneration.

From this simple example, we see the FI ruling structure: how the rules are fashioned and processed using Figure 1, and how the string is regenerated. We have seen that the inductive base was reduced to a smaller value for the example.

Non FI strings are those that are pseudo random, and in this case the antecedents of all rules will have equal length, or they grow at the same rate as the string being processsed. Examples are strings that have no pattern, and these include such things as the expansion of PI or other non-repeating, non terminating sequences (transcendental numbers). We have looked [3] at the occurrence of non-FI strings in the case where binary symbols are used as the alphabet, and their occurrence is rare. We have also shown [3] when the alphabet is richer than binary, then the occurrence of non-FI sequences is even less frequent.

\subsection{Application of a Ruling in Recognizing an Unknown Pattern}

The next example uses the Ruling shown in Table 1 resulting from the simultaneous factoring of the four strings. Each string uses the same alphabet, which is not a hard requirement. The next example uses the Ruling shown in Table 1 for the factoring of the four event strings. We also state the obvious: the more data one has about a pattern, the more accurately one can identify that pattern in unknown sequences. In order to show how rulings apply in terms of recognition of subsequences, we now provide some patterns. In this case, we are no longer concerned about regenerating the original sequences from the rulings.

Example 2. Ruling Built from Four Sequences (Simultaneous Factoring)

Suppose there are four strings designated $a, b, c$ and $d$. We have added the start symbol ' $\mathrm{S}$ ' to each string for convenience. Consider the following four strings:

$$
\begin{array}{lll}
\text { a }: \text { S2446664882443 } & \text { b : S66666254322187 } & \text { c : S666662225442217 } \\
& \text { d : S7765442218 } &
\end{array}
$$

Since we have started each string with the symbol $\mathrm{S}$, this will shorten the residuals from each string. As can be seen from the four strings the starting implicants are:

$S \rightarrow 2 ; S \rightarrow 6 ; S \rightarrow 7$; however, since this is simultaneous factoring, and these implicants are contradictory, they are not acceptable in the deterministic case. We require in simultaneous factoring that the implicants be consistent intra- as well as inter-string. The implicants $\mathrm{S} 2 \rightarrow 4$; S6 $\rightarrow$ 6; S7 $\rightarrow 7$ are consistent for all four 
sequences. The final residuals shown in (3) for each of the strings are those symbols that remain due to intra- or inter-string conflicts. The resulting table of implicants augmented with their source string, for each of the levels in the FI ruling table is given in Table 1 with the inductive base held to 2

$$
\text { a: } s \rightarrow 2 \quad \text { b: } s 6 \rightarrow 5 \quad \text { c: } 6 \rightarrow 2 \quad \text { d: } 6 \rightarrow 7
$$

Table 1. FI Ruling Table for the Strings $a, b, c$ and $d$ with an Inductive Base of 2

\begin{tabular}{|c|c|c|c|c|}
\hline Level 1 & Level 2 & Level 3 & Level 4 & Level 5 \\
\hline $\begin{array}{l}\mathrm{S} 2 \rightarrow 4(\mathrm{a}) \\
\mathrm{S} 6 \rightarrow 6(\mathrm{~b}, \mathrm{c}) \\
\mathrm{S} 7 \rightarrow 7(\mathrm{~d}) \\
82 \rightarrow 4(\mathrm{a}) \\
32 \rightarrow 2(\mathrm{~b}) \\
42 \rightarrow 2(\mathrm{~d}, \mathrm{c}) \\
24 \rightarrow 4(\mathrm{a}) \\
64 \rightarrow 8(\mathrm{a}) \\
5 \rightarrow 4(\mathrm{~b}, \mathrm{c}, \mathrm{d}) \\
46 \rightarrow 6(\mathrm{a}) \\
76 \rightarrow 5(\mathrm{~d}) \\
77 \rightarrow 6(\mathrm{~d}) \\
48 \rightarrow 8(\mathrm{a}) \\
88 \rightarrow 2(\mathrm{a})\end{array}$ & $\begin{array}{l}\mathrm{S} 2 \rightarrow 6(\mathrm{a}) \\
\mathrm{S} 6 \rightarrow 6(\mathrm{~b}, \\
\mathrm{c}) \\
\mathrm{S} 7 \rightarrow 4(\mathrm{~d}) \\
32 \rightarrow 1(\mathrm{~b}) \\
42 \rightarrow 1(\mathrm{~d}, \mathrm{c}) \\
53 \rightarrow 2(\mathrm{~b}) \\
64 \rightarrow 3(\mathrm{a}) \\
54 \rightarrow 2(\mathrm{c}) \\
74 \rightarrow 2(\mathrm{~d}) \\
26 \rightarrow 6(\mathrm{a})\end{array}$ & $\begin{array}{l}\mathrm{S} 2 \rightarrow 4(\mathrm{a}) \\
\mathrm{S} 6 \rightarrow 6(\mathrm{~b}, \mathrm{c}) \\
\mathrm{S} 7 \rightarrow 8(\mathrm{~d}) \\
3 \rightarrow 8(\mathrm{~b}) \\
54 \rightarrow 7(\mathrm{c}) \\
38 \rightarrow 7(\mathrm{~b})\end{array}$ & $\begin{array}{l}\mathrm{S} 6 \rightarrow 6(b, c) \\
\mathrm{S} 6 \rightarrow 2(b, c)\end{array}$ & $\begin{array}{l}62 \rightarrow 2(\mathrm{c}) \\
22 \rightarrow 5(\mathrm{c}) \\
65 \rightarrow 3(\mathrm{~b}) \\
25 \rightarrow 4(\mathrm{c})\end{array}$ \\
\hline
\end{tabular}

The processing that takes place in this example shows how the incoming string is matched with the known data as represented by the implicants contained within the ruling. If the antecedents match, then we can delete the consequent symbol at the end of the process for this level from the unknown string. We can match the position of this deleted symbol for later identification. The residual is the string remaining after all levels have removed the consequents matching the antecedents for the appropriate implicants in each level. The algorithm used in this matching process is called Following. As was indicated above, the residual is important as its length is a percentage of the original string suggesting an overall measure of similarity between the ruling and the string. Residuals also represent a measure of similarity based upon differences between multiple strings with the ruling acting as the adjudicator. These residuals in a sense represent the entropy of the string when the universe of experience or knowledge is contained within the ruling. 
Example 3. Recognition of Patterns in a New Sequence

Consider the following two strings formed from the new sampled events. We will designate them by $e$ and $f$. The strings are:

$$
e: s 666662278122345 \quad f: s 666662543443244666488244187
$$

The Following results are shown in Table 2, when the Ruling of Table 1 is applied to the two strings. In addition, the implicants used from Table 1 have their originating string annotation, and this annotation is copied under the bottom row of Table 2 in the line called 'Source'. In order to represent implicants with multiple sources, we encode the sources as: $(\mathrm{a})=1,(\mathrm{~b})=2,(\mathrm{c})=3,(\mathrm{~d})=4,(\mathrm{~b}, \mathrm{c})=5,(\mathrm{~d}, \mathrm{c})=6,(\mathrm{~b}, \mathrm{c}, \mathrm{d})=7$.

Table 2. Following Applied to the Two Patterns $e$ and $f$ Respectively Using Table 1

\begin{tabular}{|c|c|c|c|c|}
\hline \multicolumn{5}{|c|}{ Following } \\
\hline Level & \multicolumn{4}{|c|}{ For String $e$} \\
\hline $5:$ s 6 & \multicolumn{4}{|c|}{2278122345} \\
\hline $4:$ s 6 & & 278122 & 45 & \\
\hline $3:$ s 6 & 66 & 278122 & 45 & \\
\hline \multicolumn{5}{|c|}{$2: s 6 \quad 6662278122345$} \\
\hline \multicolumn{5}{|c|}{$1: \mathrm{s} 666662278122345$} \\
\hline Source & 5555 & 3 & & \\
\hline Level & \multicolumn{3}{|c|}{ For String $f$} & \\
\hline $5: \mathrm{s} 6$ & 25 & 344324 & 6664 & 221 \\
\hline 4: s 6 & 625 & 344324 & 6664 & 221 \\
\hline 3: s 6 & 6625 & 344324 & 6664 & 221 \\
\hline $2:$ s 6 & 66625 & 344324 & 6664 & 221 \\
\hline \multicolumn{5}{|c|}{ 1: s 6666625434432446664882221} \\
\hline Source & 555 & & & \\
\hline
\end{tabular}

From the results shown in Table 2 of the Following over $e$ and $f$ we conclude:

- Neither string matches very well the data contained in the ruling.

- $\quad$ Best matches come from a combination of $b$ and $c$ for string $e$.

- The best match for string $f$ comes initially from $b$ or $c$, and then evolves into the string of $a$.

- $\quad$ This would indicate in both sequences $e$ and $f$, that the data in the ruling is not sufficiently rich to represent the sequence, so this data should be added to the ruling.

- Modification of the ruling on the fly can be accommodated, and depending upon storage space, one can add strings at will.

- In order to control growth, the implicants can be eliminated based upon a usage criteria. A counter can be added to each rule, and when the counter drops beneath some usage limit, the rule can be deleted, or the sequence containing the rule can be deleted. 
- When a symbol has not been recognized, but the antecedent was matched, then that symbol represents a possible single symbol mutation. In this case, the system will substitute the consequent in the string, and mark the substitution, and continue recognition with the altered symbol. This is called $R e$ placement Following.

- $\quad$ For sequence substitutions, the sequence will be processed with the ruling, and the substituted sequence will not be recognized, and except for accidental similarities, replacement will not solve the similarity problem. Thus there will be a block of symbols that will remain in the residual. These symbols can be used to form a new ruling, linked to the previous ruling, so that over time, an assemblage of rulings will be formed that represent the evolution of the original string.

\section{Finite Inductive Sequences and Biometric Pattern Examples}

There are several applications that are of interest using the model that we have proposed. The first just deals with recognition of a gene from one source to another. If we extend the recognition technique described in Section 2.3, we can deal with the mutation issues within a gene. Besides the Exact Following of Section 2.3, there are several more extensions. The first allows recognition of genes that have changed from the exemplars stored in the Ruling by mutations of deletion, duplication, inversion, insertion or translocation. Consider the issue of insertion and the Skip Following process. In this process the user defines the size of a skip region, and in the gene, this involves a region that potentially has been inserted and is not in the exemplar set of the ruling. We now provide some examples to show how FI can be used in the genomic pattern matching world when various kinds of mutations are allowed.

Example 4. Mutation by insertion.

Consider sequence (5) as an exemplar sequence for the ruling content.

$$
\text { G T G A G T G G T C T T A G G T G A G T C A G T G C A G }
$$

The ruling is given in Table 3 where the levels are divided by the horizontal lines, and the Order column is the order in which the implicants were formed in processing (5).

Table 3. Ruling for Sequence (5)

\begin{tabular}{|c|c|c|c|c|c|c|c|c|c|c|c|}
\hline & ece & lent & & $q u$ & Order & & & & & & \\
\hline & & & $\mathrm{S}$ & $\mathrm{G}$ & 1 & & $\mathrm{~T}$ & A & $\mathrm{G}$ & $\mathrm{G}$ & 15 \\
\hline & & $\mathrm{S}$ & $\mathrm{G}$ & $\mathrm{T}$ & 2 & A & G & G & $\mathrm{T}$ & $\mathrm{G}$ & 17 \\
\hline & $\mathrm{S}$ & $\mathrm{G}$ & $\mathrm{T}$ & $\mathrm{G}$ & 3 & $\mathrm{G}$ & $\mathrm{G}$ & $\mathrm{T}$ & $\mathrm{G}$ & A & 18 \\
\hline & G & $\mathrm{T}$ & $\mathrm{G}$ & A & 4 & A & G & $\mathrm{T}$ & $\mathrm{C}$ & A & 22 \\
\hline & & & A & G & 5,14 & & $\mathrm{C}$ & A & $\mathrm{G}$ & $\mathrm{T}$ & 24 \\
\hline & & & & & 23,28 & $\mathrm{C}$ & A & $\mathrm{G}$ & $\mathrm{T}$ & $\mathrm{G}$ & 25 \\
\hline & G & A & $\mathrm{G}$ & $\mathrm{T}$ & 6,20 & & & $\mathrm{G}$ & $\mathrm{C}$ & $\mathrm{A}$ & 27 \\
\hline & & $\mathrm{G}$ & $\mathrm{G}$ & $\mathrm{T}$ & 9,16 & & & & $\mathrm{~S}$ & $G$ & 7 \\
\hline $\mathrm{T}$ & G & $\mathrm{G}$ & $\mathrm{T}$ & $\mathrm{C}$ & 10 & & & $\mathrm{G}$ & $\mathrm{C}$ & $\mathrm{C}$ & 26 \\
\hline G & G & $\mathrm{T}$ & $\mathrm{C}$ & $\mathrm{T}$ & 11 & & & $\mathrm{~S}$ & $\mathrm{G}$ & $\mathrm{G}$ & 8 \\
\hline & & $\mathrm{C}$ & $\mathrm{T}$ & $\mathrm{T}$ & 12 & & & $\mathrm{G}$ & $\mathrm{G}$ & $\mathrm{C}$ & 21 \\
\hline
\end{tabular}


Now consider the new sequence and their associated positions:

G T G A G T G G T C C g t a t T T A G G T G A G

LOC: $1 \begin{array}{llllllllllllllllllllllll} & 2 & 3 & 4 & 5 & 6 & 7 & 8 & 9 & 10 & 1 & 2 & 3 & 4 & 5 & 6 & 7 & 8 & 9 & 20 & 1 & 2 & 3 & 4\end{array}$

We have defined a variable called Skip in the system to permit ignoring some number of symbols in the recognition (following) process. Processing the string of (6) with the ruling of Table 3, we get the results shown in (7) when we process left to right:

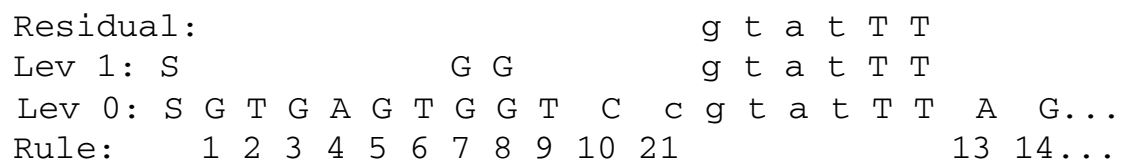

We now know that there is a difference between the ruling and the unknown sequence because of the residual. If we set the skip value to 6 for example, and starting to match from the first symbol that occurs in the residual (being the g) plus the skip value gives the next starting symbol $\mathrm{A}$ in position 18. Looking for a rule in Table 3 after 10, maintaining rule order (another parameter setting in the system), we find for the A, rules $4,13,18$, and 27. All rules are unacceptable except rule 13 due to the antecedent TT and its relative order. Since the skip value is a guess only, we need to see if backing up prior to location 18 can trim some off of the mutation region. We also note that rule 21 is not in the correct place, so removing 21, and backing up from location 18 we get the final insertion mutation candidate as: $\mathrm{c} \mathrm{g} \mathrm{t} \mathrm{a} \mathrm{t}$

The skip value being arbitrary for this example can be set by the user to an expected value, or just set to some maximal value. The only difference in reality is the length of time it requires to search backwards from the skipped location. If rule order is main-tained, then the processing will be much faster, since all previous rules used up to the first location where a nucleotide appears in the residual will not need to be considered.

\section{Example 5. SNP Processing}

Suppose we have an exemplar sequence around a SNP loci where an allele exists as shown in Table 4. The problem is to determine the frequency of SNPs over a set of loci to allow likelihood patterns for determining both missing nucleotides as well as determining the predominate nucleotide in a chromosome.

Table 4. SNP Patterns from Individuals (Rows) and from a Chromosome (Column)

\begin{tabular}{llllll} 
& M & & M & & M \\
\hline 85 & CC & 22 & CG & 45 & CG \\
91 & CC & 29 & CC & 41 & CC \\
93 & CG & 34 & CG & 57 & CC \\
94 & CG & 48 & CC & 61 & CG \\
83 & CC & 55 & CG & 27 & CC
\end{tabular}


The surrounding symbols to these alleles are identical in the chromosome and suppose that we define the precursor symbols to be CACC with the two possible sequences for $\mathrm{M}$ to be CACCC or CACCG with a frequency of eight and seven. If one doesn't know where the SNP happens to reside, then one can follow one gene in one chromosome from the ruling of a gene in the exemplar chromosome with the SKIP value set to one, or one can use Replacement Following and keep track of the loci and type of replacements done.

Consider the rules derived for the string CACCC and CACCG. The rules, permitting non-determinism are shown in (8). The current, internal form of the Ruling is a Finite State Machine (FSM), and to add non-determinism, we simply add another state for the additional consequent, and a counter to record the number of times each rule was used. For the last two rules of (8), a single rule of the form $\mathrm{CC} \rightarrow$ $\mathrm{C}(8) / \mathrm{G}(7)$ would suffice in the FSM where the 8 and 7 are the frequency counts for occurrences.

$$
\mathrm{S} \rightarrow \mathrm{C}, \mathrm{SC} \rightarrow \mathrm{A}, \mathrm{A} \rightarrow \mathrm{C}, \mathrm{AC} \rightarrow \mathrm{C}, \mathrm{CC} \rightarrow \mathrm{C}(8), \mathrm{CC} \rightarrow \mathrm{G}(7)
$$

For Replacement following, suppose the exemplar sequence was the first five rules implying that the symbol pair CC always produced a $\mathrm{C}$. With replacement suppose we found the string CACCG, the result would be to delete the $\mathrm{G}$ and replace it with the $\mathrm{C}$. If counting is turned on, then the number for each of the replaced symbols would be counted. Additionally a position marker can be output to identify to the location of each replacement.

Example 6. Trace for Following

Suppose we have the ruling of Table 3, and the unknown sequence shown in (9). Here the sequence is only similar to that used as the exemplar in Table 3. One of the things which the system will do is provide a trace of the implicant usage.

SGTTGGCCCGTGGTTT TA G G T G A T TGG TGCA G

(9a) represents Level 0 implicant application to the sequence of (9), and (9b) represents the residual from Level 0 . (9b) and (9c) represent the processing from Level 1. The final residual is given in (10).

$$
\begin{array}{lllll}
\text { S T G } & \text { C T TGG T T T } & \text { A T G }
\end{array}
$$

The residual contains seventeen symbols implying that fifteen symbols were deleted. The more interesting observation is the subsequence of symbols beginning with rule number 13 and running consecutively through rule number 18 in a cluster with no skipped nucleotides indicating the presence of a subsequence of potential interest. 


\section{Conclusion}

The need for exact matching in a fast algorithm is important for continued research and development for genomic understanding and disease characterization. We have implemented the algorithms for both the Factoring and Following, and they operate in their basic mode in near $\mathrm{O}(\mathrm{n})$ [4] time where $\mathrm{n}$ is the length of the sequence to follow. With the newest version of the algorithm based upon a direct addressing scheme for the antecedents in an implicant, we have yet to actually obtain real performance data. It should be clear based upon how the ruling is structured, that one can achieve faster processing by placing levels of a ruling on several independent processors and connect the processors in a pipeline configuration. This will provide a simple, pipelined, parallel algorithm with speed of $\mathrm{O}(\mathrm{n} / \mathrm{p})$ where $\mathrm{p}$ is the number of processors. Alternatively, one can place the entire ruling on each of several processors in a cluster or network, and then supplying overlapping (by the inductive base) subsets of the unknown string to each processor. Again the process complexity becomes $\mathrm{O}(\mathrm{n} / \mathrm{p})$. In order to achieve these speeds, we first used a finite state machine model indicated earlier as the ruling structure. Storage space has an upper limit of the inductive base $*_{n}$ again where $\mathrm{n}$ is the length of the string to be factored.

With the new algorithm we are using a large amount of internal memory to hold the ruling, and the following process is simply a direct access into that storage structure. This has limited the inductive base to approximately three codons, but access for any nucleotide would be then, if found ruling-levels/2, and if not found then simply the number of levels in the ruling. Since this number is small, the complexity for following would be at most $\mathrm{O}(\mathrm{n} *$ number-of-levels-in-Ruling), which is $\mathrm{O}(\mathrm{n} * \mathrm{k})$ or just $\mathrm{O}(\mathrm{n})$. This is the more important time constraint, due to the fact that a ruling is created once and used many times in identifying patterns.

\section{References}

1. Altschul, S.F., Gish, W., Miller, W., Myers, E.W., Lipman, D.J.: Basic local alignment search tool. Journal of Molecular Biology 215(3), 403-410 (1990)

2. Buckingham, S.D.: Scientific software: seeing the SNP's between us. Nature Methods 5, 903-908 (2008)

3. Case, J., Fisher, P.S.: Long Term Memory Modules. Bulletin of Mathematical Biology 46(2) (1984)

4. Das, S., Fisher, P.S., Zhang, H.: Efficient Parallel Algorithms for Pattern Recognition. In: Proceedings of Twenty-sixth Hawaii International Conference in Systems Sciences (January 1993)

5. Pearson, W.: Flexible sequence similarity searching with FASTA3 program package. In: Misener, S., Krawety, S.A. (eds.) Bioinformatics Methods and Protocols, pp. 185-219. Humana Press, Inc., Totowa (2000)

6. Pearson, W.R., Lipman, D.J.: Improved tools for biological sequence comparison. Proc. National Academy of Science 85, 2444-2448 (1988)

7. Smith, T.F., Waterman, M.S.: Identification of Common Molecular Subsequences. Journal of Molecular Biology 147, 195-197 (1981) 
8. Uberbacher, E.: Computing the Genome, http: / /www. ornl.gov/info/ornlreview/v30n3-4/genome.htm

9. Wang, G.Y., Fisher, P.: Knowledge Acquisition: Neural Network Learning. In: Data Mining and Knowledge Discovery: Theory, Tools, and Technology. II SPIE, vol. 4057, pp. 117-128 (2000)

10. Xu, Y., Mural, R.J., Einstein, J.R., Shah, M., Uberbacher, E.C.: GRAIL: A Multi-Agent Neural Network System for Gene Identification. Proceedings of The IEEE 84(10), 1544 1552 (1996) 